\title{
Neural correlates of rehabilitation program with robot-assisted intensive therapy in one case of Holmes tremor
}

Bénédicte PONTIER MD, MSc ${ }^{(1,2,3)}$, Anna SONTHEIMER PhD ${ }^{(1,2)}$, Jérôme COSTE PhD ${ }^{(1,2)}$, Youssef El OUADIH MD, MSc ${ }^{(1,2)}$, Anne-Marie BRIANCON MD ${ }^{(4)}$, Guilhem MILLET OTR/L ${ }^{(5)}$, Eric PANTERA MD, MSc ${ }^{(3)}$, Catherine SARRET MD, PhD ${ }^{(2)}$, JeanJacques LEMAIRE MD, PhD ${ }^{(1,2)}$.

(1) Service de neurochirurgie, CHU de Clermont-Ferrand, Clermont-Ferrand, F63000, France ;

(2) Université Clermont Auvergne, CNRS, SIGMA Clermont, Clermont-Ferrand, F63000, France ;

(3) Centre de Médecine Physique et Réadaptation, Pionsat, F- 63330, France ;

(4) Centre Hospitalier Étienne Clémentel, Enval, F- 63530, France ;

(5) SAMSAH de LADAPT Auvergne, Clermont-Ferrand, F-63000, France.

Corresponding author: Bénédicte PONTIER, service de Neurochirurgie, CHU de Clermont-Ferrand, 58 rue Montalembert, 63003 Clermont-Ferrand cedex, France bpontier@chuclermontferrand.fr

Tel $+33(0) 473752166$

\section{Keywords}

Holmes tremor, functional MRI, diffusion tensor imaging, rehabilitation. 
Holmes tremor $(\mathrm{HT})$ is a rare movement disorder characterized by lowfrequency tremor, inferior to $5 \mathrm{~Hz}$, at rest and during postural and intentional conditions (1). It severely alters the functional abilities. The pathophysiology of tremor is unknown. However, dysfunctions of cortico-ponto-cerebello-thalamo-cortical loops were hypothesized by Bucy in 1944 and confirmed since notably in HT $(2,3)$, involving contralateral sensorimotor and prefrontal cortices and the ipsilateral cerebellum. Treatments of HT include levodopa, dopamine agonists, anticholinergics, propranolol, primidone, levetiracetam, more recently botulinum toxin and deep brain stimulation, with variable and unpredictable responses (4). Hence we built a personalized rehabilitation program with intensive robotic rehabilitation, as proposed after stroke (5), associated to classical human-guided rehabilitation, in one case of HT. The lesions were scrutinized using structural magnetic resonance imaging (MRI). Both clinical effects and functional MRI (fMRI) activations were studied before and after rehabilitation, willing to explore underlying neural correlates.

\section{Clinical picture}

A 23-year-old man, right-handed, developed a severe tremor of the right upper limb two years after a traumatic brain injury (Glasgow coma score $=6$ ) with diffuse axonal lesions. The whole upper limb was involved, and the tremor extended at a lesser degree to the trunk and head. The frequency was low and it was present at rest and during postural and intentional conditions. The tremor was severe, involving both the proximal and distal segments of the upper limb, affecting all daily activities. Joint mobility, strength and muscle tone were normal. Previously he did not benefit of escitalopram, topiramate, baclofen and levodopa treatments. The patient also suffered of a discrete left contralateral motor hemiparesis and a slight cognitive impairment.

\section{MRI lesions}

MRI lesion load was noticed from T1- and T2- weighted MRI, Fluid-Attenuated Inversion Recovery and Susceptibility Weighted Imaging sequences. We observed sequelae of diffuse axonal lesions notably in the frontal lobes, associated with cortical and subcortical atrophy. Deep situated focal lesions were found in the left thalamus and the midbrain (figure 1): a left hemi mesencephalic zone showing hemosiderin deposit, involving notably the substantia nigra, the red nucleus and the tegmental reticular formation; a right brain peduncle atrophy; a marked atrophy of the superior and anterior portion of the left thalamus associated with hemosiderin deposit. Colorcoded fractional anisotropy maps computed from Diffusion Tensor Imaging (DTI, 20 directions) and DTI fiber tracking (Iplan 3.0; BrainLab, Munich, Germany) revealed details of white matter lesions in the tegmentum and the brain peduncle, affecting: corticopontine and corticospinal tracts, notably the pyramidal tract centered on superior limb fibers, and brachium conjunctivum (superior cerebellar peduncle) and lemniscal fascicles. 

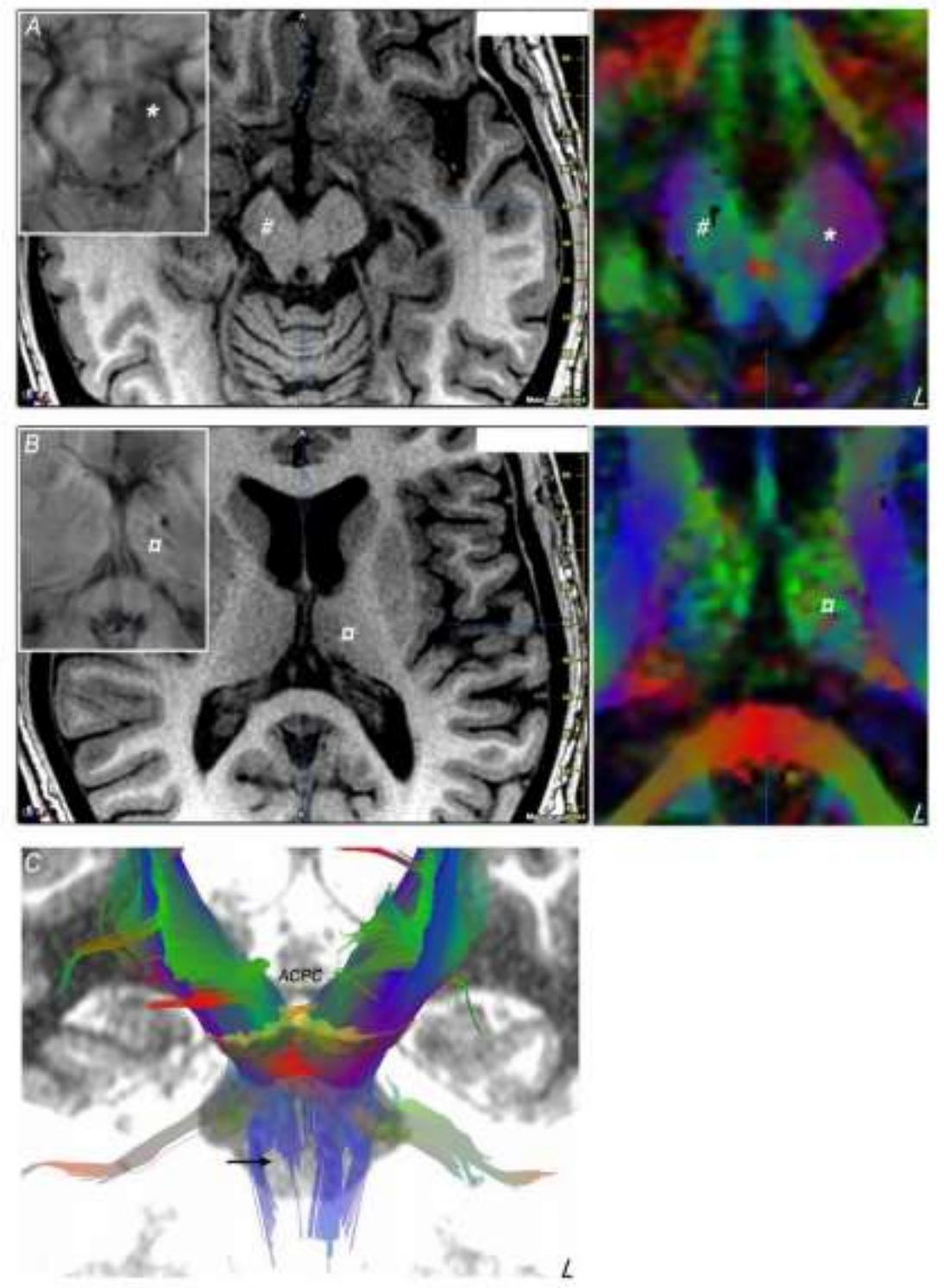

Figure 1: Mesencephalic $(A)$ and thalamic $(B)$ lesions showed on axial MRI slices (left, T1- weighted image; inlay susceptibility weighted image; right, color-coded fractional anisotropy map) parallel to the anterior commissure - posterior commissure (ACPC) line: left hemi mesencephalic area showing hemosiderin deposit (*); right brain peduncle atrophy (\#); atrophy of the superior portion of the left thalamus associated with hemosiderin deposit (a). Fascicles going through the mesencephalon ( $C$, frontal view), with the defect (arrow) of right projection fibers (the ACPC line is displayed on the midline)

\section{Rehabilitation program}

The program was built to improve the accuracy of motor movements: intensive work on single or two-hand coordination, speed and precision activities with a physiotherapist and an occupational therapist. An intensive robotic rehabilitative management consisted of four hours of elbow and shoulder training per day during three continuous weeks. The robot was the exoskeleton "Armeo®Spring" (Hocoma, USA) which embraced the whole arm, from shoulder to hand, and cancelled the tremor, 
allowing targeted and repeated exercises of the limb with biofeedback. The patient agreed with the full program.

\section{Clinical and imaging assessments}

Clinical and $\mathrm{fMRI}$ data were recorded one week before and fifteen days after the rehabilitation program. Clinical evaluations included Functional Independence Measure (6), Box and Block Test (assessment of gross manual dexterity (7)), Minnesota Manual Dexterity Test (assessment of rapid eye-hand coordination and arm-hand dexterity). The functional impact on daily activities was also assessed, each item being rated from 0 , unable to do the activity, to 3 , able to do the activity effortlessly: drinking with a glass, transferring water from one glass to another, cutting an apple, lacing shoes, buttoning, writing, drawing a spiral, zipping.

fMRI with a motor task consisted of rapid and repeated prono-supination movements of the forearm, combined with alternating hand opening and closing. The task was performed for the right and the left upper limb before and after rehabilitation. We extracted fMRI bloodoxygen-level dependent signal clusters statistically different (positive; $p<0.05$, family-wise error corrected; SPM 12, Wellcome Center for Human Neuroimaging, UCL, London, UK) during the task versus at rest condition, and following the rehabilitation, between the right and left upper limb tasks.

\section{Clinical and fMRI modifications following the rehabilitation program}

The patient showed a significant reduction of tremor severity: the time required to move small objects (Minnesota Test) was reduced by $44 \mathrm{~s}$ (14'36 to 13'52), the number of blocks transported from one compartment to another in two minutes (Box and Block Test) was slightly better (75 versus 71 ). Manual skills improved in writing, cutting an apple, drawing a spiral and zipping (overall scores, 3 and 7, respectively before and after rehabilitation). Writing slowly with the right hand became possible whereas it was not possible before rehabilitation. However, no difference was found in the Functional Independence Measure (score = 125; before and after)

For the tremoric right upper limb, activation clusters were located before rehabilitation in the left sensorimotor cortex and supplementary motor area and the right cerebellum, and after rehabilitation in the left sensorimotor cortex and the right and left cerebellum, and in the temporal lobes (T3 anterior and posterior, bilaterally; left T1, T4 posterior). For the left upper limb, activation clusters were located, before rehabilitation, in the right and left sensorimotor cortex, the left cerebellum, and the right anterior and posterior region of $\mathrm{T} 1$, and after rehabilitation in the left and right sensorimotor cortices, the cerebellum, and lateral sulci. Only the right upper limb task versus the left, after rehabilitation, elicited significant activation, and this bilaterally in frontopolar cortices. The overall results are figure 2. 


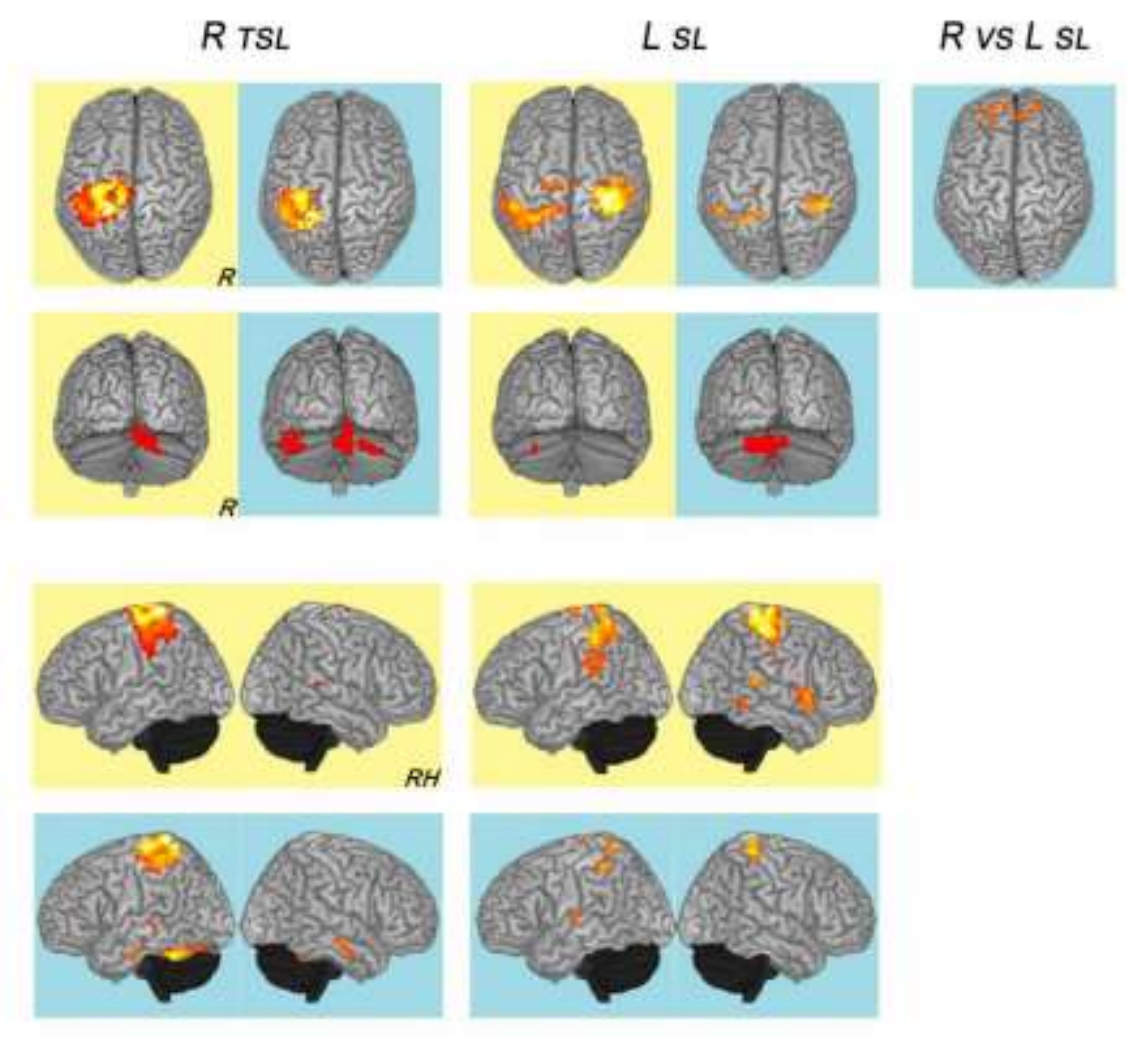

Figure 2: fMRI activations (superior, posterior and lateral views; right, $R$; $R$ hemisphere, $\mathrm{RH}$ ) following motor task of prono-supination movements of the forearm, combined with alternating hand opening and closing, before (yellow) and after (blue) the rehabilitation program: right tremoric superior limb (RTSL) and left superior limb (LSL) tasks versus at rest; right superior limb versus left superior limb activation ( $R$ vs $L S L$ ).

\section{Discussion}

The HT patient, fulfilling the criteria of HT $(1,3)$, benefited from the intensive personalized rehabilitation program alleviating the tremor and enabling to perform precise movements, although the Functional Independence Measure remained stable. The contribution of the robot-assisted intensive therapy cannot be inferred, as it was coupled with human-guided assistance.

Lesions underlying the clinical presentation were detailed: tremor of the right superior limb extended to the trunk and head, contralateral to lesions of the left thalamus and the left mesencephalon; left motor hemiparesis contralateral to the atrophy of right corticospinal tract, and slight cognitive impairment due to diffuse hemispheric lesions. Diffuse axonal lesions in frontal white matter could have worsened the movement disorder.

The fMRI data, before and after rehabilitation, confirmed the involvement of contralateral sensorimotor and premotor cortices and ipsilateral cerebellum. Interestingly we observed ipsilateral activations only during left upper limb tasks, before and after rehabilitation, which could be explained by reorganization of the predominance of one hemisphere over the other during movement (8). The 
rehabilitation also seems to engage fronto-temporal cortices that could reflect an increased cognitive and attentional load $(9,10)$, and the implication of other dimensions of movements (11). Indeed, post rehabilitation bitemporal activations were elicited by the task of the right tremoric superior limb, and at a lesser extent by the left superior limb. In parallel the fronto-polar activation of regions was essentially supported by the left dominant (right-handed patient) hemisphere.

In conclusion, although not specific to $\mathrm{HT}$, our findings contribute to the knowledge of neural correlates of rehabilitation program in tremor, notably for further neurofeedback training (12). Moreover it also shows that neurorehabilitation processes can be explored from a neural point of view and should beneficiate of structural and functional approaches. Mean and long term effects should be studied. Finally, the coupling between clinical and imaging data could help the design of personalized healthcare.

We have no conflict of interest and no financial support for this research.

\section{References}

1. Bhatia KP, Bain P, Bajaj N, Elble RJ, Hallett M, Louis ED, et al. Consensus Statement on the classification of tremors. from the task force on tremor of the International Parkinson and Movement Disorder Society. Mov Disord. 2018;33(1):75- 87.

2. Muthuraman M, Raethjen J, Koirala N, Anwar AR, Mideksa KG, Elble R, et al. Cerebello-cortical network fingerprints differ between essential, Parkinson's and mimicked tremors. Brain J Neurol. 01 2018;141(6):1770- 81.

3. Joutsa J, Shih LC, Fox MD. Mapping holmes tremor circuit using the human brain connectome. Ann Neurol. déc 2019;86(6):812- 20.

4. Espinoza Martinez JA, Arango GJ, Fonoff ET, Reithmeier T, Escobar OA, Furlanetti $L$, et al. Deep brain stimulation of the globus pallidus internus or ventralis intermedius nucleus of thalamus for Holmes tremor. Neurosurg Rev. oct 2015;38(4):753- 63.

5. Mehrholz J, Hädrich A, Platz T, Kugler J, Pohl M. Electromechanical and robotassisted arm training for improving generic activities of daily living, arm function, and arm muscle strength after stroke. Cochrane Database Syst Rev. 13 juin 2012;(6):CD006876.

6. Pretz CR, Kean J, Heinemann AW, Kozlowski AJ, Bode RK, Gebhardt E. A Multidimensional Rasch Analysis of the Functional Independence Measure Based on the National Institute on Disability, Independent Living, and Rehabilitation Research Traumatic Brain Injury Model Systems National Database. J Neurotrauma. 15 2016;33(14):1358- 62.

7. Chen H-M, Chen CC, Hsueh I-P, Huang S-L, Hsieh C-L. Test-retest reproducibility and smallest real difference of 5 hand function tests in patients with stroke. Neurorehabil Neural Repair. juin 2009;23(5):435- 40. 
8. Verstynen T, Diedrichsen J, Albert N, Aparicio P, Ivry RB. Ipsilateral motor cortex activity during unimanual hand movements relates to task complexity. J Neurophysiol. mars 2005;93(3):1209- 22.

9. Sakurada T, Goto A, Tetsuka M, Nakajima T, Morita M, Yamamoto S-I, et al. Prefrontal activity predicts individual differences in optimal attentional strategy for preventing motor performance decline: a functional near-infrared spectroscopy study. Neurophotonics. avr 2019;6(2):025012.

10. Bocková $M$, Chládek J, Jurák $P$, Halámek J, Rektor I. Executive functions processed in the frontal and lateral temporal cortices: intracerebral study. Clin Neurophysiol. déc 2007;118(12):2625- 36.

11. Lingnau A, Downing PE. The lateral occipitotemporal cortex in action. Trends Cogn Sci. mai 2015;19(5):268- 77.

12. Auer T, Dewiputri WI, Frahm J, Schweizer R. Higher-order Brain Areas Associated with Real-time Functional MRI Neurofeedback Training of the Somato-motor Cortex. Neuroscience. 15 2018;378:22- 33.

\section{Authors' Roles}

1. Research Project: A. Conception. B. Organization. C. Execution; 2. Statistical Analysis: A. Design. B. Execution. C. Review and Critique; 3. Manuscript: A. Writing of the first draft. B. Review and Critique.

B.P.: 1A, 1B, 1C. 3A A.S.: 1A. 1C. 3B J.C.: 3B B.J.: 1A AM.B.: 1B, 1C G.M.: 1C E.P.:3B C.S.:3B JJ.L.: 1A, 2A, 3B 